\title{
OPEN No evidence for basigin/CD147 as a direct SARS-CoV-2 spike binding receptor
}

\author{
Jarrod Shilts ${ }^{1}$, Thomas W. M. Crozier $\mathbb{D}^{2}$, Edward J. D. Greenwood², Paul J. Lehner ${ }^{2}{ }^{2}$ \& \\ Gavin J. Wright $1,3 \bowtie$
}

The spike protein of SARS-CoV-2 is known to enable viral invasion into human cells through direct binding to host receptors including ACE2. An alternate entry receptor for the virus was recently proposed to be basigin/CD147. These early studies have already prompted a clinical trial and multiple published hypotheses speculating on the role of this host receptor in viral infection and pathogenesis. Here, we report that we are unable to find evidence supporting the role of basigin as a putative spike binding receptor. Recombinant forms of the SARS-CoV-2 spike do not interact with basigin expressed on the surface of human cells, and by using specialized assays tailored to detect receptor interactions as weak or weaker than the proposed basigin-spike binding, we report no evidence for a direct interaction between the viral spike protein to either of the two common isoforms of basigin. Finally, removing basigin from the surface of human lung epithelial cells by CRISPR/Cas9 results in no change in their susceptibility to SARS-CoV-2 infection. Given the pressing need for clarity on which viral targets may lead to promising therapeutics, we present these findings to allow more informed decisions about the translational relevance of this putative mechanism in the race to understand and treat COVID-19.

The sudden emergence of SARS-CoV-2 in late 2019 has demanded extensive research be directed to resolve the many uncharted aspects of this previously-unknown virus. One essential question is what host factors the virus uses to recognize and invade human cells. SARS-CoV-2, as with other members of the coronavirus family, invades host cells using the large trimeric spike proteins on its surface. A series of studies published within the first few months of the COVID-19 pandemic independently confirmed that the same angiotensin-converting enzyme 2 (ACE2) receptor that was found to mediate SARS spike binding to human cells also mediates SARS-CoV-2 binding to human cells ${ }^{1-3}$. However, for previous coronaviruses closely related to SARS-CoV-2 including SARS and MERS, multiple different host receptors have been described with roles facilitating viral invasion ${ }^{4-7}$, making it plausible that additional interaction partners for the SARS-CoV-2 spike may remain undiscovered. Among the most prominent claims for an alternate SARS-CoV-2 host receptor comes from a report identifying basigin (CD147) as a binding partner for the SARS-CoV-2 spike protein with functional significance in viral invasion ${ }^{8}$. Based on a previously-published indirect interaction between the SARS spike protein and cyclophilin A for which basigin appeared to be involved, basigin was subsequently found to directly bind the spike SARS-CoV-2 spike protein with reasonably high affinity (equilibrium dissociation constant of $185 \mathrm{nM}$, compared to 5-20 nM reported for the similarly high-affinity spike-ACE2 binding ${ }^{2,9}$ ). Direct binding between the SARS-CoV-2 spike "receptor-binding region" of the S1 domain and basigin was demonstrated in those reports by co-immunoprecipitation, surface plasmon resonance, and enzyme-linked immunosorbent assays (ELISAs).

Notably, the original finding that basigin is a possible alternative SARS-CoV-2 receptor has already translated into an open-label clinical trial of a humanized therapeutic monoclonal antibody against basigin, meplazumab, which reported striking improvements in COVID-19 patients treated with antibody ${ }^{10}$. Basigin represents an attractive medical target because therapeutic agents have already been developed that target basigin based on basigin's previously-established role as an essential host receptor for invasion of the malaria parasite Plasmodium falciparum $^{11,12}$. The claim that basigin acts as a host receptor for SARS-CoV-2 has already featured in published articles discussing the prioritization of therapeutics ${ }^{13}$, and has been the subject of published analyses looking at basigin expression on the assumption that it serves as a viral entry factor ${ }^{14-16}$.

${ }^{1}$ Cell Surface Signalling Laboratory, Wellcome Sanger Institute, Cambridge, UK. ${ }^{2}$ Cambridge Institute of Therapeutic Immunology and Infectious Disease, University of Cambridge, Cambridge, UK. ${ }^{3}$ Department of Biology, York Biomedical Research Institute, Hull York Medical School, University of York, Wentworth Way, York, UK.『email: js44@sanger.ac.uk; gw2@sanger.ac.uk 
We sought to validate the interaction between the SARS-CoV-2 spike protein and human basigin after observing that the result had not yet been reproduced despite intense interest in the interaction's proposed consequences. Using a variety of sensitive approaches for detecting binding interactions and validated reagents, we were unable to find any supporting evidence for a direct interaction of the SARS-CoV-2 spike protein with basigin. Consistent with this, we found no functional role for BSG in the infection of a human lung cell line with SARS-CoV-2. Based on our findings, we encourage caution in approaches aimed at addressing the current pandemic caused by SARS-CoV-2 which are rooted in the assumption that basigin acts as a viral recognition receptor without further evidence.

\section{Results}

We first investigated whether basigin (BSG) expressed on the surface of human cell lines could bind the spike protein of SARS-CoV-2. The previous reports of this interaction had not performed any binding experiments on full-length basigin displayed on the surface of cells ${ }^{8}$. First, we synthesized constructs to recombinantly express the spike protein of SARS-CoV-2. We emulated the published designs of spike constructs previously determined to be folded and functional ${ }^{17}$. Using a mammalian HEK293 expression system to increase the chances that structurally-critical post-translational modifications would be preserved ${ }^{18}$, we produced both the full extracellular domain of the spike protein, and the S1 domain of the spike that mediates all known receptor binding events (Fig. 1a). When HEK293 cells were transiently transfected with cDNA overexpression plasmids for ACE2, the transfected cells became strongly stained by fluorescent tetramers of spike protein in either S1 or full forms (Fig. 1b). However, no similar gain of binding was observed with HEK293 cells transfected with BSG cDNA (Fig. S1). BSG transfection led to significant upregulation of cell-surface BSG (Welch's t-test of BSG transfected vs mock-transfected cells $p=0.01$ ), yet we also noted that these HEK293 cell lines express BSG at high levels even without cDNA overexpression (Fig. 1c). Despite this, spike protein tetramers had no detectable background staining of our HEK293 cells without ACE2 in either our experiment or similar experiments reported with SARS-CoV-2 and HEK293 cells ${ }^{19-21}$.

Next, we sought to leverage the high sensitivity of direct biochemical binding assays to determine if these methods could detect any traces of basigin binding. We have previously expressed the ectodomain of the basigin receptor in a functionally active form and used it to discover pathogen ligands including Plasmodium falciparum RH5 ${ }^{11,22,23}$. In a HEK293 human cell line, we expressed recombinant forms of the extracellular domains of both the canonical isoform of basigin (BSG) that contains two Ig-like domains and an alternate isoform which contains an additional Ig-like domain (BSG-long) (Fig. 2a). To confirm our recombinant constructs were folded and biochemically active, we probed the basigin constructs with three different monoclonal antibodies known to bind native basigin at the cell surface ${ }^{12,24}$ in enzyme-linked immunosorbent assays (ELISAs). All antibodies specifically bound to both of our recombinant basigin isoforms but not a negative control construct of recombinant rat Cd4 tag (Fig. 2b). The protein epitopes recognized by these antibodies retained their conformation in recombinant basigin but less so in basigin that is denatured by heat and reducing agent, with each antibody having between twofold to $>$ tenfold reduced immunoreactivity after treatment (Fig. 2c). To determine significance, we fit loglogistic dose-response models to each protein and antibody combination that had at least two replicates ${ }^{25}$, with Bonferroni-corrected p-values ranging from 0.02 (BSG and MEM-M6/1) to <0.0001 (all others) when comparing denatured and control curves by F-tests.

With the functionality of our constructs quality-tested, we performed a plate-based binding assay ${ }^{26}$ that uses the avidity gains of multimerized proteins to detect even highly transient protein-protein interactions ${ }^{27}$. The SARS-CoV-2 spike proteins and ACE2 gave clear binding signals in both binding orientations as plate-bound baits and reporter-linked preys, yet no signals were observed for either BSG isoform against either spike construct (Fig. 3a). By contrast the known interaction between BSG and Plasmodium falciparum RH5 was readily detected, as was a control low-affinity interaction between human CD200 and CD200R. Notably, these interactions have similar or even weaker affinity than reported for the BSG-spike interaction ${ }^{11,28}$. Finally, in response to recent reports of a mutation in the SARS-CoV-2 spike that is rapidly displacing the reference sequence ${ }^{29,30}$, we also checked whether the D614G variant of the spike could bind BSG; again, we could not detect any interaction (Fig. $3 \mathrm{~b}$ ). In all configurations the signal from BSG binding spike protein was indistinguishable from the background of non-interacting protein pairs and significantly below the known interaction pairs (Welch's t-test of ACE2-spike interactions vs BSG-spike interactions $p=0.0002$ ) (Fig. 3c).

While these results strongly suggested that the spike protein of SARS-CoV-2 cannot directly bind basigin as an entry receptor, to account for the possibility of basigin acting through less direct routes in vivo, we tested whether basigin has any functional role in viral infection of a commonly used lung epithelial cell line $(\mathrm{CaLu}-3)^{1,31}$. We transduced Cas9-expressing CaLu-3 cells with single guide (sg) RNAs targeting ACE2, BSG, and, as a negative control against a similarly abundant receptor, beta-2-microglobulin (B2M) to decrease cell surface MHC class I expression ${ }^{32}$. We achieved a strong decrease in cell surface expression of each protein encoded by these genes (Fig. S2). We then challenged each cell line with an infectious strain of SARS-CoV-2 across a range of doses, measuring which cells the virus entered by staining against the viral nucleocapsid protein (Fig. 4a). Targeting of the known ACE2 receptor by CRISPR-Cas9 led to a near-complete loss of infectivity (two-way ANOVA of ACE2 infection vs B2M control Bonferroni-corrected $p$-value $<0.001$ ), despite these cells retaining very high expression of BSG (Fig. 4b). In contrast, genetic removal of BSG had no significant effect on infectivity, with similarly high rates of viral entry seen compared to the B2M control (two-way ANOVA of BSG infection vs B2M infection Bonferroni-corrected $p$-value $>0.90$ ) and to the parental CaLu-3 cells having no genes knocked out. 
a

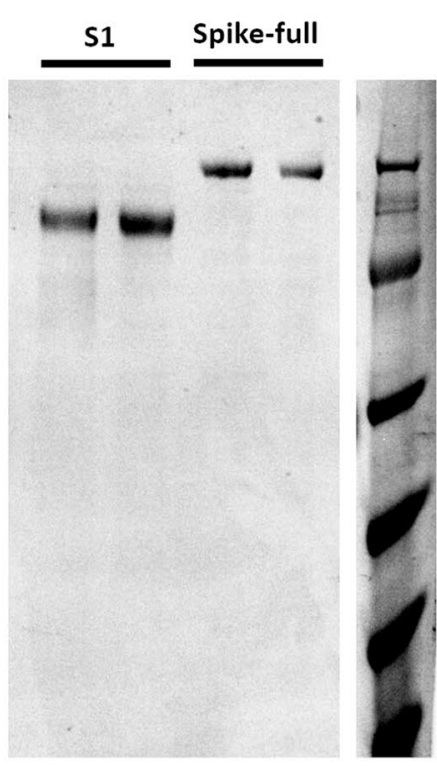

C b

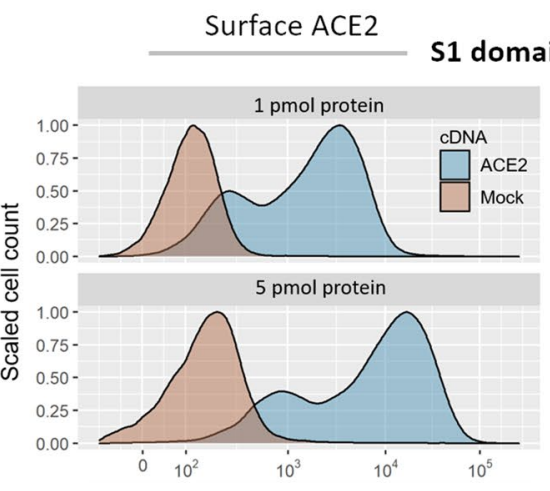

Surface BSG

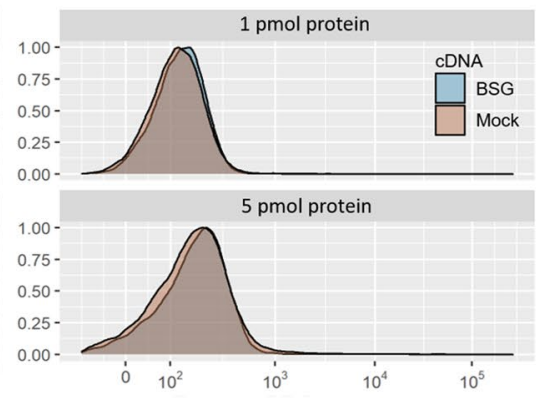

Full trimeric spike binding

51

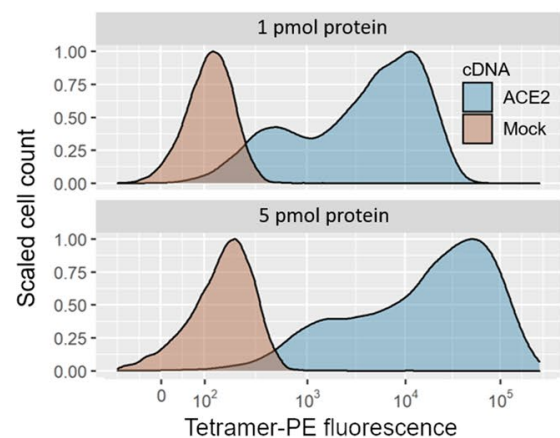

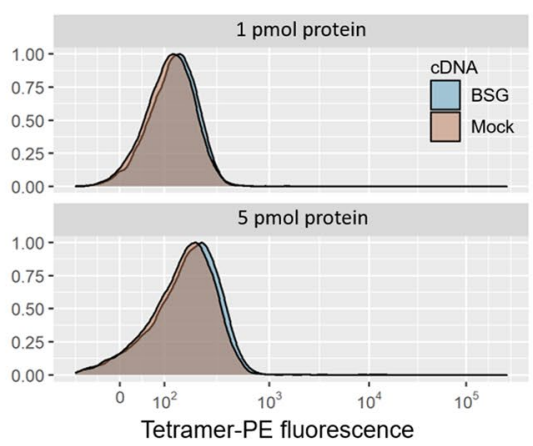
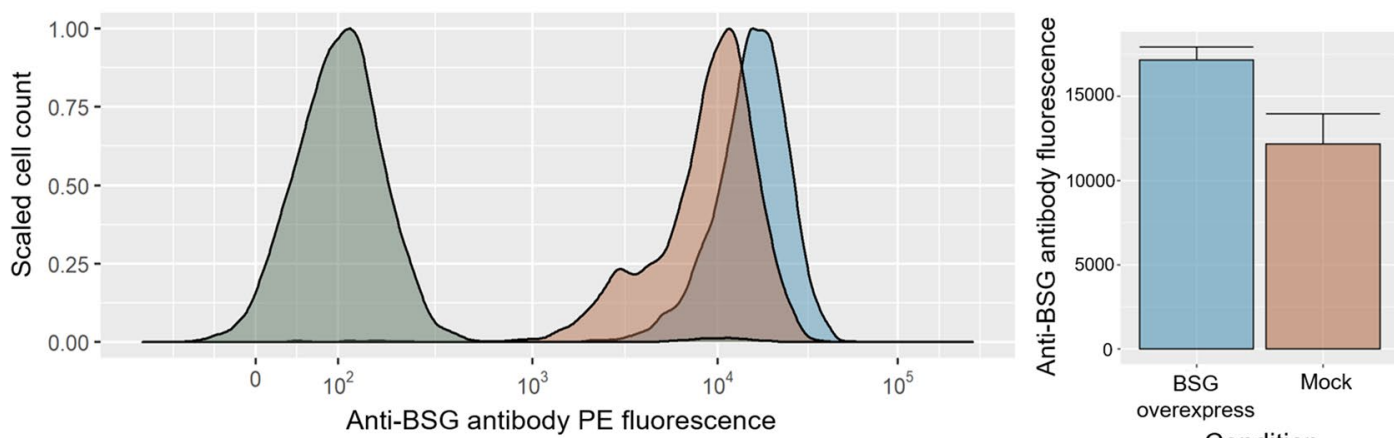

Condition

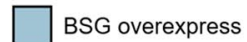

Condition

Figure 1. Gain of SARS-CoV-2 spike binding activity on human cells over-expressing ACE2 but not BSG. (a) Expression and purification of the S1 domain and full ectodomain of the SARS-CoV-2 spike protein produced in human cell lines. Two independent preparations of purified spike were resolved by SDS-PAGE under reducing conditions and stained with Coomassie blue dye. (b) Cells transfected with cDNAs encoding ACE2 but not BSG bind highly avid fluorescent SARS-CoV-2 spike tetramers. Flow cytometry fluorescence distributions of cells stained with tetramers composed of biotinylated spike protein either using the S1 domain (top panels) or the entire ectodomain (lower panels) clustered around phycoerythrin-conjugated streptavidin. The stained HEK293 cells were transfected with cDNA to overexpress either ACE2 (left) or BSG (right). Mock-transfected cells are shown in red. Similar behavior to the data shown was observed in three separate tests. (c) Transfection with BSG cDNA leads to upregulation of cell-surface BSG. Surface basigin levels on HEK293 cells labeled with an anti-human BSG monoclonal antibody. BSG levels are compared to a negative control of secondary-antibody only. Mean antibody fluorescence intensities are summarized in the adjacent bar graph, with error bars showing standard deviations $(n=2-4)$.

\section{Discussion}

Identifying the host receptors which a virus can recognize is an important step in mechanistically explaining viral infection, and can offer insight in a virus' cellular tropism and factors influencing susceptibility. Despite the importance of determining precisely which entry receptors SARS-CoV-2 uses to infect human cells, there remains considerable uncertainty amid multiple claims of several viral receptors with variable qualities of data to support these assertions ${ }^{8,33-36}$. We investigated one of the most prominent claims, that human BSG acts as an alternate receptor for the virus to interact with, which has been the topic of several studies, news and review articles, and a clinical trial ${ }^{10,14,15,37-42}$. Our access to established tools and reagents from previous work studying BSG's role as a host receptor in Plasmodium infection allowed us to rapidly investigate BSG as a SARS-CoV-2 receptor. Despite validating the functionality of all our reagents, we were unable to detect any binding in biochemical or cell-based 


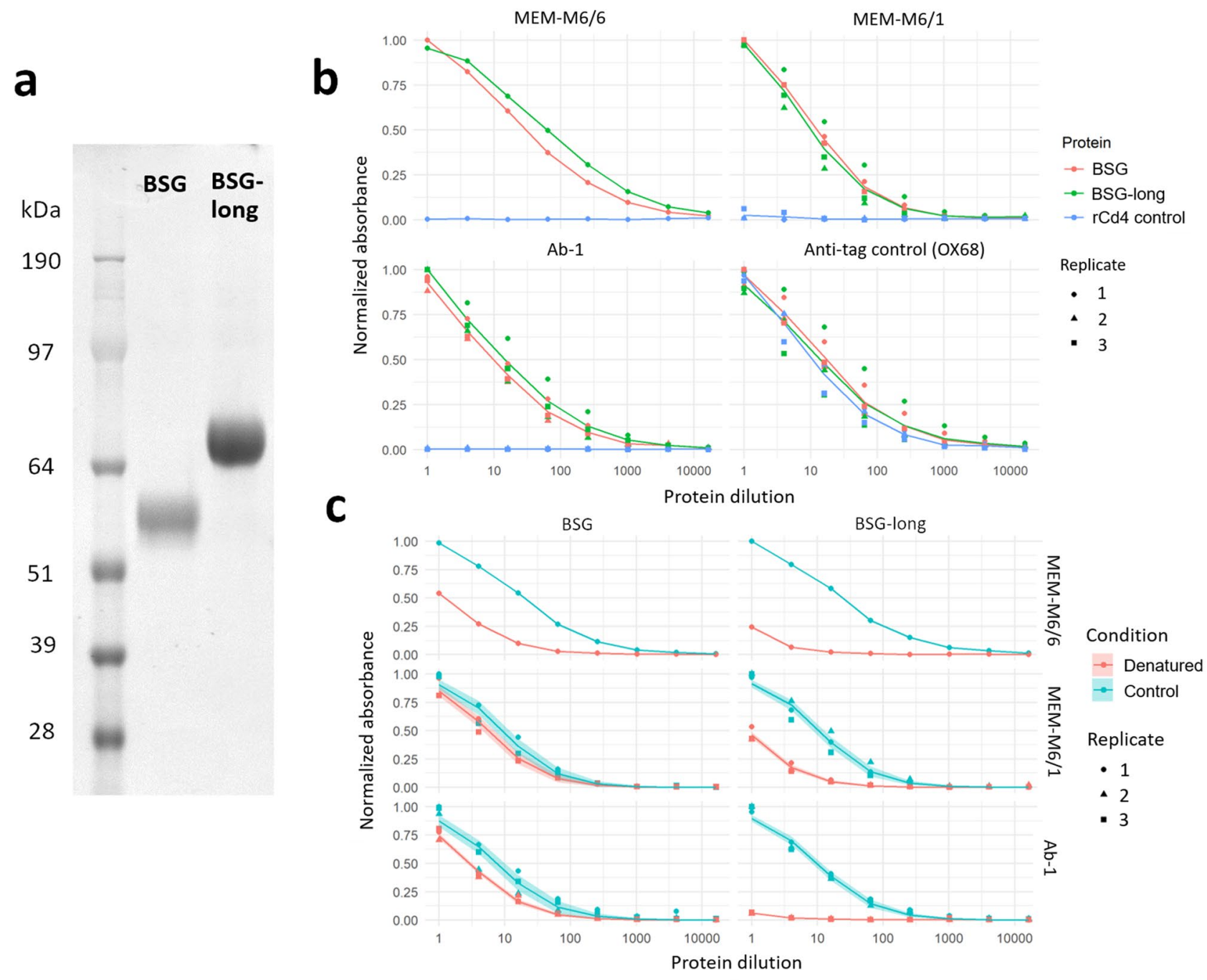

Figure 2. Basigin expressed as recombinant protein ectodomains retain their biochemical activity. (a) Expression and purification of two and three Ig-like domain isoforms of basigin. Proteins were resolved under reducing conditions by SDS-PAGE and stained with a Coomassie dye (b). Recombinant basigin but not control proteins are recognized by anti-basigin monoclonal antibodies. ELISA dilution series of BSG and BSG-long recognized by three different monoclonal antibodies, and a control OX68 antibody against their tags. A negative control of a recombinant Cd4 tag is included for each antibody. (c) Recombinant basigin retains folded conformation of epitopes recognized by three different monoclonal antibodies. ELISA dilution curves comparing unmodified basigin to protein treated with heat and a reducing agent. Three replicates were performed for all ELISA curves except MEM-M6/6, for which only a single trial was done. Dose response curve model fit lines are superimposed on the data points, with shading indicating the $95 \%$ confidence bounds of the models.

assays for either common BSG isoform or either configuration or allele of the SARS-CoV-2 spike protein. Further, in viral infection assays with authentic SARS-CoV-2 in lung cell lines, we observed no role of BSG in infection.

BSG is highly expressed on many cell types throughout the body, including activated lymphocytes and red blood cells, forming the basis of the Ok blood grouping system ${ }^{43}$. Notably, SARS-CoV-2 has not been found to enter red blood cells ${ }^{44}$. However, the possibility that BSG could act as an accessory binding receptor for the virus has been speculated in several publications to possibly explain in part the link between SARS-CoV-2 infection and hematological symptoms in patients ${ }^{39,40,45,46}$. Our data suggest this hypothesis should be treated cautiously. Similarly, if our negative findings are replicated, it would necessitate a re-interpretation of the clinical trial involving injections of anti-BSG monoclonal antibodies, as any patient benefit would be more likely explained by alternative hypotheses such as immune modulation as opposed to direct blockage of viral invasion through BSG. Hypotheses relying on BSG binding to explain viral tropism may also need closer reconsideration ${ }^{14}$.

A recent genomic study investigating variants in genes reported to be linked to SARS-CoV-2 infection failed to find evidence of BSG variants enriched in COVID-19 patients, despite identifying associations with more established viral entry factors such as TMPRSS2 ${ }^{47}$. Combined with our own infection tests in lung cells with BSG genetically ablated, we see no evidence supporting a direct role for BSG in viral infection. While our results all 

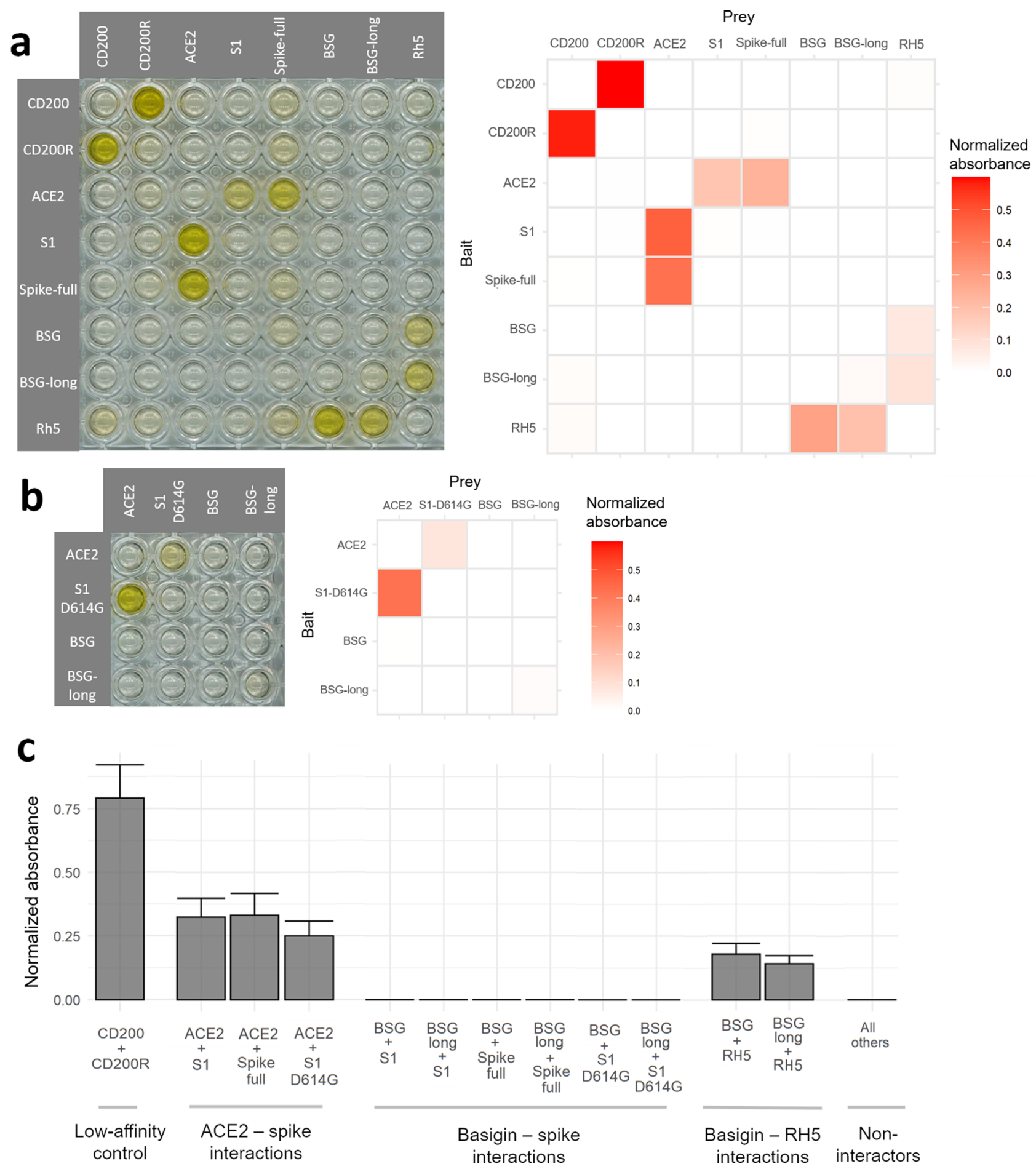

Figure 3. Sensitive assays designed to detect extracellular protein interactions do not detect a direct interaction between human basigin and the SARS-CoV-2 spike protein. (a) No signs of spike-basigin binding in an aviditybased protein interaction assay systematically testing a matrix of recombinant baits immobilized to streptavidincoated plates (rows) against preys clustered around HRP-conjugated streptavidin (columns). A photograph of a representative assay plate (left) is shown alongside background-corrected absorbance values averaged across two replicates. (b) The emerging D614G mutant variant of the SARS-CoV-2 spike also does not bind basigin. Binding matrix including the common D614G variant of the SARS-CoV-2 spike protein instead of the reference sequence. (c) Spike protein binding to basigin is consistently undetectable compared to other control interactions. Binding signals were averaged across bait and prey orientations for known interacting protein pairs, the basigin-spike pairs, and all other pairs. Error bars represent standard deviation from the mean $(n=2$, except "All others" $\mathrm{n}=58$ ). 


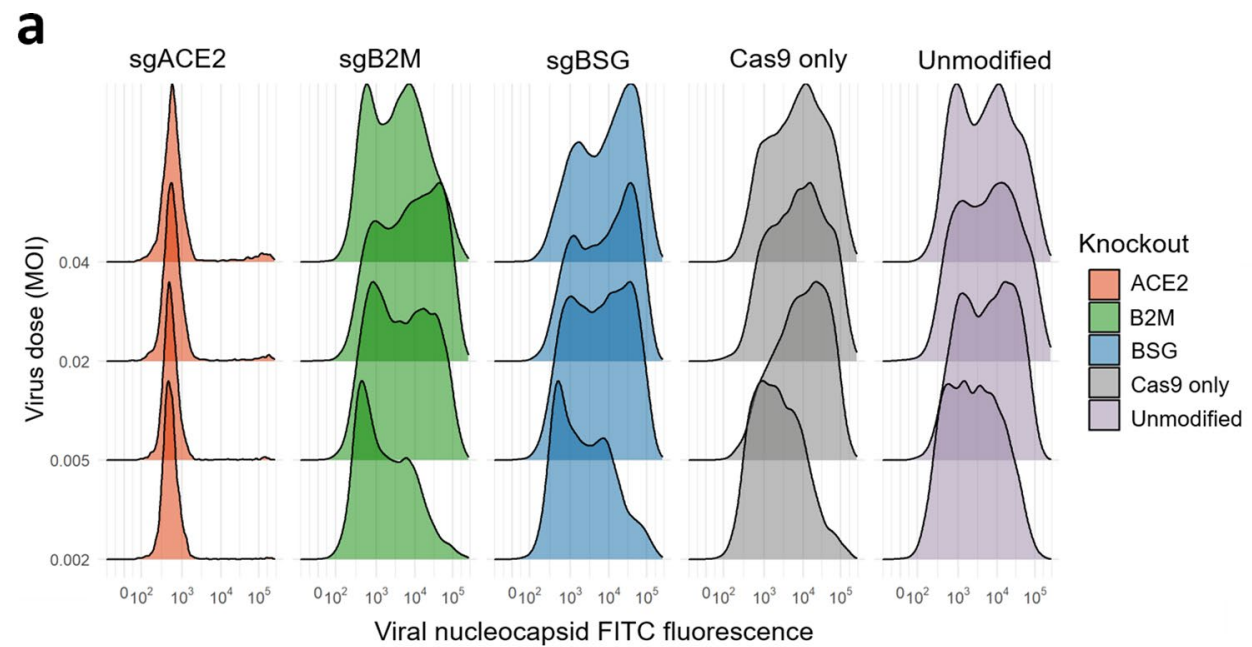

b

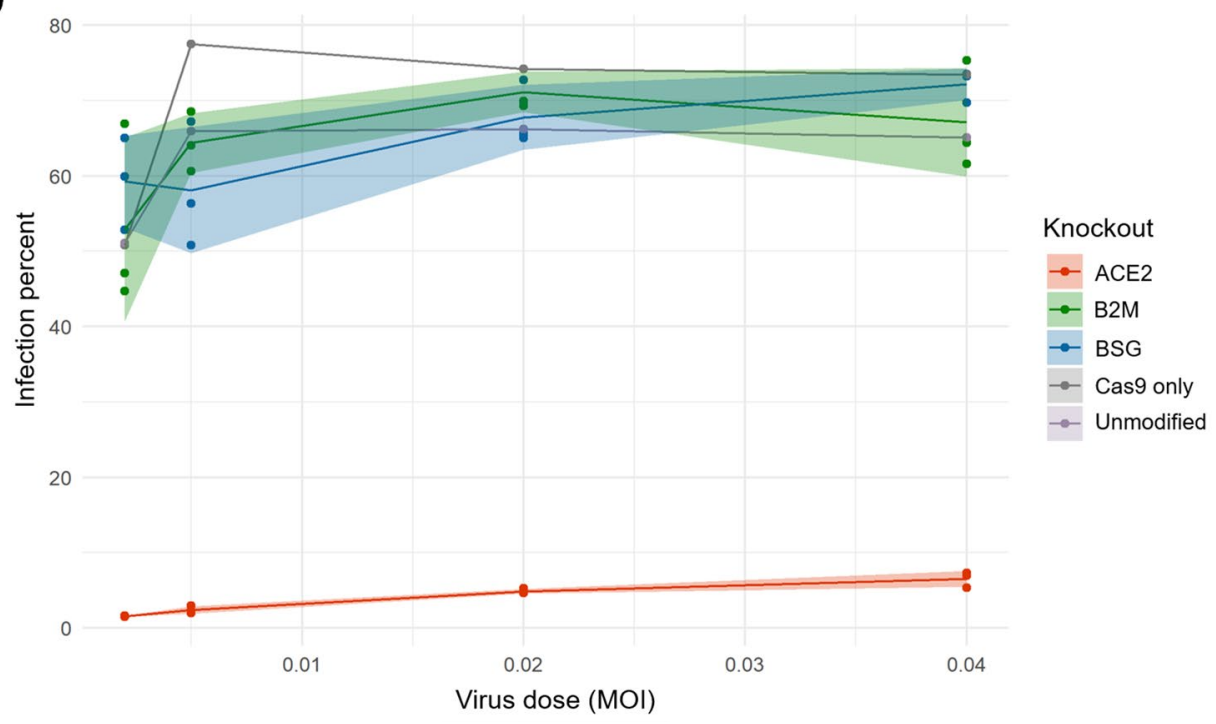

Figure 4. Knockdown of basigin by CRISPR-Cas9 in a lung cell line has no effect on susceptibility to SARSCoV-2 infection. (a) Knockout of the known viral entry receptor ACE2 blocks viral infection in CaLu-3 cells, but not single guide (sgRNA) knockouts of basigin or negative control MHC class I receptors. Representative flow-cytometry profiles of SARS-CoV-2 nucleocapsid protein in CaLu-3 cells $48 \mathrm{~h}$ post-infection. (b) Lung cells with cell-surface basigin knocked down have no significant change in rates of viral entry and SARS-CoV-2 infection. Three replicates were performed of all 3 knockout conditions. Shading represents standard deviation from the mean percent of cells infected.

point to the lack of a direct role for BSG, as mentioned above, BSG could still have some biological relevance through indirect routes ${ }^{48}$. For example, through its previously-described role in the development of the immune system, BSG could indirectly influence COVID-19 clinical progression ${ }^{49}$.

Although our findings are negative, they nevertheless carry important potential implications to both our understanding of the basic biology of SARS-CoV-2 and efforts to translate knowledge of the virus' host receptors into therapeutics. We encourage greater study in confirming the mechanisms that have been proposed, not just for BSG but also for the multiple other putative viral receptors, so as to resolve the uncertainty around whether SARS-CoV-2 utilizes any receptors beyond ACE2 during infection.

\section{Materials and methods}

Expression construct design. cDNA expression constructs were taken from a previously-assembled library of full-length human cDNAs in human expression vectors ${ }^{50}$. The BSG construct was cloned from a copy (Origene \#RG203894) of the canonical 2-domain isoform of BSG (NM_198589.1), while the ACE2 cDNA (NM_021804.2) was expressed from a similar expression vector utilizing a CMV promoter (Geneocopia \#EX-U1285-M02). A small amount of MCT4 (NM_001042422.1), which is known to facilitate BSG surface expression $^{51}$, was co-transfected along with BSG at a 1:4 ratio to achieve significant BSG overexpression. The recombinant human BSG ectodomain constructs have been previously described ${ }^{11,23}$ and span from M1-L206 
(BSG) or M1-L322 (BSG-long). The extracellular domain truncations for the SARS-CoV-2 spike proteins have also been previously published ${ }^{17}$. The S1 domain was defined as spanning Q14-Y647, while the full spike ectomain spanned Q14-K1211. The endogenous viral signal peptide was replaced with an efficient mouse antibody signal peptide ${ }^{52}$. As previously described, the full spike ectodomain was mutated at its polybasic protease cleavage site (682-685 RRAR to SGAG), had a proline stabilizing mutation introduced (986-987 kV to PP), and to mimic the natural trimerized structure of the spike had a foldon trimerization domain introduced at its C-terminus. The ACE2 ectodomain spanned M1-S740, retaining its endogenous signal peptide.

Recombinant protein expression and purification. Human embryonic kidney (HEK)-293E cells were transiently transfected with polyethylenimine as previously described ${ }^{53,54}$. Per $100 \mathrm{~mL}$ of cells, $50 \mu \mathrm{g}$ of plasmid was transfected along with $1 \mu \mathrm{g}$ of a plasmid encoding the biotin-ligase BirA to direct biotinylation of the recombinant proteins ${ }^{55}$. Cells were grown in Freestyle Media (Life Technologies \#12338018) supplemented with $100 \mu \mathrm{M}$ D-biotin (Sigma \#2031). Human proteins were incubated for $120 \mathrm{~h}$ at $37^{\circ} \mathrm{C}$, while spike proteins were shifted to $34^{\circ} \mathrm{C}$ and supplemented with $0.5 \%(\mathrm{~m} / \mathrm{v})$ tryptone N1 (OrganoTechnie \# 19553) 24 h post-transfection and incubated a further $96 \mathrm{~h}$ based on a published spike-specific optimized protocol ${ }^{56}$. After incubation, cell culture supernatants were harvested and passed through $0.22 \mu \mathrm{m}$ filters. Purification was done using nickelnitrilotriacetic acid (Ni-NTA) resins (Thermo Scientific \#88221) that were pre-washed for 10 min in 2 washes of $25 \mathrm{mM}$ imidazole (Sigma \#I2399) phosphate buffer. Supernatants were mixed with pre-washed Ni-NTA resin overnight at $4{ }^{\circ} \mathrm{C}$, then washed three times with $25 \mathrm{mM}$ imidazole phosphate buffer before eluting in $200 \mathrm{mM}$ imidazole buffer. Purified proteins were analyzed on $4-12 \%$ gradient Bis-Tris gels (Invitrogen \#NP0329) following denaturation for $10 \mathrm{~min}$ at $80{ }^{\circ} \mathrm{C}$ in NuPAGE sample buffer (Invitrogen \#NP0007, \#NP0004). Across experimental replicates, independent batches of protein were used, with the exception of BSG and BSG-long for which a single batch was quality-tested and used in all subsequent experiments.

Flow cytometry and tetramer binding assays. To generate transfected cells overexpressing cellsurface receptors, human embryonic kidney (HEK)-293E cells were seeded one day prior to transfection at a density of $2.5 \times 10^{5}$ cells per $\mathrm{mL}$ in Freestyle Media (Life Technologies \#12338018) supplemented with $10 \%$ heatinactivated fetal bovine serum (FBS). Cells were transiently transfected with polyethylenimine as previously described $^{4,22}$, except with double the ratio of DNA to cells. Then $48 \mathrm{~h}$ after transfection, cells had culture media aspirated and were resuspended in $1 \mu \mathrm{M}$ DAPI (Biolegend \#422801) and incubated on ice for 5 min. Cells were then stained in u-bottom 96-well plates (Greiner \#650161) with recombinant protein tetramers conjugated to phycoerythrin (PE). Tetramers were prepared by mixing 1 or 5 pmol of biotinylated protein monomer with 0.25 to $1.25 \mathrm{pmol}$ of streptavidin-PE (Biolegend \#405245) respectively and incubating for $2 \mathrm{~h}$ at room temperature. Cells were spun down and resuspended in $100 \mu \mathrm{L}$ of tetramer in a solution of $1 \%$ bovine serum albumin (BSA) and phosphate buffered-saline (PBS) supplemented with calcium and magnesium ions (Gibco \#14040133). Cells and tetramers were incubated on ice for $45 \mathrm{~min}$, washed in cold PBS, and finally resuspended in the same $1 \%$ BSA PBS solution. Antibody staining was performed with a similar procedure, except during the first 30 min on ice cells were incubated with $30 \mu \mathrm{g} / \mathrm{mL}$ of monoclonal Ab-1 anti-BSG antibody ${ }^{12}$, then resuspended in 1:500 anti-human IgG antibody conjugated to Cy3 (Sigma \#C2571). Fluorescence staining was measured by a BD Fortessa flow cytometer.

Monoclonal antibody ELISAs. Streptavidin-coated 96-well plates (Nunc \#436014) were pre-washed in $175 \mu \mathrm{L}$ hepes-buffered saline (HBS) with $0.1 \%$ tween-20 (HBS-T), then blocked in $2 \%$ ( $\mathrm{m} / \mathrm{v}$ ) bovine serum albumin (BSA, Sigma \#A9647) in HBS for $1 \mathrm{~h}$ at room temperature. In a separate 96-well plate, a 1:4 dilution series of biotinylated BSG or control protein was prepared in $2 \%$ BSA HBS, then $100 \mu \mathrm{L}$ of the protein dilution transferred to the blocked streptavidin-coated 96-well plate. In the experiments to determine the protein's sensitivity to heat and reduction treatment, one half of the protein sample was denatured by heating at $80{ }^{\circ} \mathrm{C}$ for $10 \mathrm{~min}$ in the presence of $5 \%$ beta-mercaptoethanol. After capturing protein for $1 \mathrm{~h}$ at room temperature, plates were washed three times with $150 \mu \mathrm{L}$ HSB-T. Anti-human basigin monoclonal antibodies were added at the following concentrations: $1.7 \mu \mathrm{g} / \mathrm{mL} \mathrm{Ab}-1$ (Zenonos et al., formerly known as ch6D9, recognizing the IgC2 domain) ${ }^{12}$, $2.2 \mu \mathrm{g} / \mathrm{mL}$ MEM-M6/1 (Abcam \#ab666), and $1.3 \mu \mathrm{g} / \mathrm{mL}$ MEM-M6/6 (Abcam \#ab119114) ${ }^{24}$. A control mouse anti-rat Cd4 domain 3+4 monoclonal antibody (OX68) against the tags of our recombinant proteins was used at a $1.6 \mu \mathrm{g} / \mathrm{mL}$ concentration. After $1 \mathrm{~h}$ of incubation with the primary antibody and three HBS-T washes, secondary antibody was added as 1:7000 donkey anti-human IgG (Abcam \#ab102407) for ch6D9 and for all other antibodies as 1:3500 goat anti-mouse IgG (Sigma \#A9316). Both secondary antibodies were conjugated to alkaline phosphatase. After $45 \mathrm{~min}$ of incubation with the secondary antibody, the plates were washed again three times with HBS-T. A substrate of $60 \mu \mathrm{L} 2 \mathrm{mg} / \mathrm{mL}$ para-Nitrophenylphosphate (Sigma \#P4744) in diethanolamine buffer was added to each well to develop signal over $30 \mathrm{~min}$. Absorbance was measured on a Tecan plate reader at $405 \mathrm{~nm}$.

Avidity-based binding assays. Biotinylated recombinant proteins were tetramerized around streptavidin-HRP (Pierce \#21130) for $1 \mathrm{~h}$ at room temperature to form reporter-linked preys. Per well of the assay plate, $0.1 \mathrm{pmol}$ of recombinant monomer were added to $0.025 \mathrm{pmol}$ of streptavidin to create a highly avid binding reagent. Streptavidin-coated 96-well plates (Nunc \#436014) were pre-washed in $175 \mu \mathrm{L}$ hepes-buffered saline (HBS) with $0.1 \%$ tween-20 (HBS-T), then blocked in $2 \%(\mathrm{~m} / \mathrm{v})$ bovine serum albumin (BSA, Sigma \#A9647) in HBS for $1 \mathrm{~h}$ at room temperature. Biotinylated baits were captured by adding 0.1 pmol of purified protein diluted in $2 \%$ BSA to each well of the plate. After incubating the baits for $2 \mathrm{~h}$ at $4{ }^{\circ} \mathrm{C}$, plates were washed three times with $150 \mu \mathrm{L}$ HBS-T. The pre-formed tetrameric preys were then added and the plate incubated for $1 \mathrm{~h}$ at room 
temperature. The plate was finally washed twice in $150 \mu \mathrm{L}$ HBS-T and once in $150 \mu \mathrm{L}$ HBS before adding $60 \mu \mathrm{L}$ 3,3',5,5'-Tetramethylbenzidine (TMB) substrate (Sigma \#T0440). After developing signal for 15 min at room temperature, the reaction was halted by the addition of $0.25 \mathrm{M} \mathrm{HCl}$. Absorbance was measured on a Tecan plate reader at $405 \mathrm{~nm}$.

CaLu-3 genome editing and validation. CaLu-3 cells were cultured in Minimum Essential Media (MEM) with 10\% FCS, 1 mM sodium pyruvate, MEM non-essential amino acids and GlutaMAX. CaLu-3 were transduced with lentivirus containing FLAG-NLS-Cas9 cloned into pHRSIN.pSFFV MCS(+) pSV40-Blast vector, and selected with blasticidin at a concentration of $10 \mu \mathrm{g} / \mathrm{mL}$. Single-guide RNAs (sgRNAs) against ACE2 and B2M were cloned into pKLV.U6-gRNA-pGK-Puro-P2A-BFP vector (Addgene \#50946). sgRNA lentiviruses were transduced into CaLu-Cas9 cells and selected with puromycin at a concentration of $2 \mu \mathrm{g} / \mathrm{mL}$. Following puromycin selection of sgRNA expressing CaLu-Cas $9, \sim 5 \times 10^{6}$ trypsinized cells were harvested for cell-surface staining. Cells were pelleted and resuspended in a solution of 5\% FCS in PBS with either 3.75 pmol full spike ectodomain tetramer conjugated to AF647, or a mixture of $1 \mu \mathrm{g} / \mathrm{mL}$ anti-MHC-I mouse monoclonal antibody (W6/32) and anti-BSG human monoclonal antibody (Ab-1) and incubated on ice for $1 \mathrm{~h}$. Cells stained with primary antibodies were washed and stained on ice with goat anti-mouse conjugated to Alexa Fluor-568 (Thermo Fisher \#A11031) and goat anti-human conjugated to Alexa Fluor-647 (Thermo Fisher \#A21445) secondary antibodies at a dilution of 1:2000 for $30 \mathrm{~min}$. Cells were washed and resuspended in FCS PBS solution prior to analysis on a BD Fortessa flow cytometer.

SARS-CoV-2 infection assays. The SARS-CoV-2 virus used in this study is the clinical isolate named "SARS-CoV-2/human/Liverpool/REMRQ0001/2020"31,57. In total, the stock used was passaged three times in VeroE6 cells, once in CaCo- 2 cells and once in CaLu-3 cells. Viral titre was determined by $50 \%$ tissue culture infectious dose $\left(\mathrm{TCID}_{50}\right)$ in Huh7-ACE2 cells. For viral infection assays $5 \times 10^{4} \mathrm{CaLu}-3$ cells were plated per well of a 24 -well tissue culture dish $72 \mathrm{~h}$ prior to addition of SARS-CoV-2 virus at the indicated MOI. At $48 \mathrm{~h}$ postinfection cells were washed with PBS, dissociated with TrypLE, and fixed in $4 \%$ formaldehyde for 15 min. Fixed cells were washed and incubated for $15 \mathrm{~min}$ at room temperature in Perm/Wash buffer (BD \#554723). Permeabilized cells were pelleted, stained for $15 \mathrm{~min}$ at room temperature in $100 \mu \mathrm{L}$ of sheep anti-SARS-CoV-2 nucleocapsid antibody (MRC-PPU, DA114) at a concentration of $0.7 \mu \mathrm{g} / \mathrm{mL}$, washed and incubated in $100 \mu \mathrm{L}$ AF488 donkey anti-sheep (Jackson ImmunoResearch \#713-545-147) at a concentration of $2 \mu \mathrm{g} / \mathrm{mL}$ for $15 \mathrm{~min}$ at room temperature. Stained cells were pelleted and fluorescence staining analysed on a BD Fortessa flow cytometer.

Data processing. For flow cytometry experiments, measurement events for analysis were gated on live singlet cells (based on DAPI and forward and side scatter profiles) using FlowJo version 9. No compensation was done because only a single fluorochrome was used for cell staining. Cytometry data was visualized using the CytoML package ${ }^{58}$ in $\mathrm{R}$ version 3.6.1. For plate-based experiments, raw absorbance values had background subtracted. Background for ELISAs was defined as the minimum absorbance of any well on the measured plate, and background for binding assays was defined as the median absorbance of each respective tetrameric prey. For better comparisons across replicates, these corrected absorbances were rescaled by min-max normalization so that the maximum absorbance on that replicate's entire plate is defined as 1 . Statistics on binding assay data were calculated using the t.test function in the $\mathrm{R}$ base stats package (version 3.6.1). Two-way ANOVAs on viral infection data were likewise done in $\mathrm{R}$ with the aov function for an additive model of viral nucleocapsid-positive cells from viral dose and knockout condition. Statistics on ELISA data were calculated by performing an F-test comparing a two-parameter log-logistic dose response model fitted to the ELISA data to a null model where both the denatured and control protein conditions were assumed to be identical. Model fitting and statistical procedures were done using the drc package in $\mathrm{R}$ as previously described ${ }^{25}$. Data is available upon request.

Received: 30 July 2020; Accepted: 20 December 2020

Published online: 11 January 2021

\section{References}

1. Hoffmann, M. et al. SARS-CoV-2 cell entry depends on ACE2 and TMPRSS2 and is blocked by a clinically proven protease inhibitor. Cell https://doi.org/10.1016/j.cell.2020.02.052 (2020).

2. Lan, J. et al. Structure of the SARS-CoV-2 spike receptor-binding domain bound to the ACE2 receptor. Nature https://doi. org/10.1038/s41586-020-2180-5 (2020).

3. Wrapp, D. et al. Cryo-EM structure of the 2019-nCoV spike in the prefusion conformation. Science 367, 1260-1263 (2020).

4. Chan, C.-M. et al. Carcinoembryonic antigen-related cell adhesion molecule 5 is an important surface attachment factor that facilitates entry of middle east respiratory syndrome coronavirus. J. Virol. 90, 9114-9127 (2016).

5. Chu, H. et al. Middle East respiratory syndrome coronavirus and bat coronavirus HKU9 both can utilize GRP78 for attachment onto host cells. J. Biol. Chem. 293, 11709-11726 (2018).

6. Jeffers, S. A. et al. CD209L (L-SIGN) is a receptor for severe acute respiratory syndrome coronavirus. Proc. Natl. Acad. Sci. U. S. A. 101, 15748-15753 (2004).

7. Marzi, A. et al. DC-SIGN and DC-SIGNR interact with the glycoprotein of marburg virus and the S protein of severe acute respiratory syndrome coronavirus. J. Virol. 78, 12090-12095 (2004).

8. Wang, K. et al. SARS-CoV-2 invades host cells via a novel route: CD147-spike protein. bioRxiv 2020.03.14.988345. https://doi. org/10.1101/2020.03.14.988345. (2020).

9. Walls, A. C. et al. Structure, function, and antigenicity of the SARS-CoV-2 spike glycoprotein. Cell 181, 281-292.e6 (2020). 
10. Bian, H. et al. Meplazumab treats COVID-19 pneumonia: an open-labelled, concurrent controlled add-on clinical trial. medRxiv 2020.03.21.20040691. https://doi.org/10.1101/2020.03.21.20040691. (2020).

11. Crosnier, C. et al. Basigin is a receptor essential for erythrocyte invasion by Plasmodium falciparum. Nature 480, 534-537 (2011).

12. Zenonos, Z. A. et al. Basigin is a druggable target for host-oriented antimalarial interventions. J. Exp. Med. 212, 1145-1151 (2015).

13. Liu, C. \& Zhu, D. Cyclophilin A and CD147: novel therapeutic targets for the treatment of COVID-19. Med. Drug Discov. https:// doi.org/10.1016/j.medidd.2020.100056 (2020).

14. Leonardi, A., Rosani, U. \& Brun, P. Ocular surface expression of SARS-CoV-2 receptors. Ocular Immunol. Inflammation 0, 1-4 (2020).

15. Radzikowska, U. et al. Distribution of ACE2, CD147, CD26 and other SARS-CoV-2 associated molecules in tissues and immune cells in health and in asthma, COPD, obesity, hypertension, and COVID-19 risk factors. Allergy https://doi.org/10.1111/all.14429 (2020).

16. Aguiar, J. A. et al. Gene expression and in situ protein profiling of candidate SARS-CoV-2 receptors in human airway epithelial cells and lung tissue. Eur. Respir. J. https://doi.org/10.1183/13993003.01123-2020 (2020).

17. Wang, Q. et al. Structural and functional basis of SARS-CoV-2 entry by using human ACE2. Cell 181, 894-904.e9 (2020).

18. Wood, L. \& Wright, G. J. Approaches to identify extracellular receptor-ligand interactions. Curr. Opin. Struct. Biol. 56, 28-36 (2019).

19. Zang, R. et al. TMPRSS2 and TMPRSS4 promote SARS-CoV-2 infection of human small intestinal enterocytes. Sci. Immunol. 5, $562(2020)$.

20. Ou, X. et al. Characterization of spike glycoprotein of SARS-CoV-2 on virus entry and its immune cross-reactivity with SARS-CoV. Nat. Commun. 11, 1620 (2020).

21. Bojkova, D. et al. SARS-CoV-2 and SARS-CoV differ in their cell tropism and drug sensitivity profiles. bioRxiv 2020.04.03.024257. https://doi.org/10.1101/2020.04.03.024257. (2020).

22. Galaway, F., Yu, R., Constantinou, A., Prugnolle, F. \& Wright, G. J. Resurrection of the ancestral RH5 invasion ligand provides a molecular explanation for the origin of P. falciparum malaria in humans. PLOS Biol. 17, e3000490 (2019).

23. Wanaguru, M., Liu, W., Hahn, B. H., Rayner, J. C. \& Wright, G. J. RH5-Basigin interaction plays a major role in the host tropism of Plasmodium falciparum. PNAS 110, 20735-20740 (2013).

24. Koch, C. et al. T cell activation-associated epitopes of CD147 in regulation of the T cell response, and their definition by antibody affinity and antigen density. Int. Immunol. 11, 777-786 (1999).

25. Ritz, C., Baty, F., Streibig, J. C. \& Gerhard, D. Dose-response analysis using R. PLoS ONE 10, e0146021 (2015).

26. Dundas, K. et al. Alpha-v-containing integrins are host receptors for the Plasmodium falciparum sporozoite surface protein, TRAP. Proc. Natl. Acad. Sci. U.S.A. 115, 4477-4482 (2018).

27. Bushell, K. M., Söllner, C., Schuster-Boeckler, B., Bateman, A. \& Wright, G. J. Large-scale screening for novel low-affinity extracellular protein interactions. Genome Res. 18, 622-630 (2008).

28. Wright, G. J. et al. Lymphoid/neuronal cell surface OX2 glycoprotein recognizes a novel receptor on macrophages implicated in the control of their function. Immunity 13, 233-242 (2000).

29. Korber, B. et al. Spike mutation pipeline reveals the emergence of a more transmissible form of SARS-CoV-2. bioRxiv 2020.04.29.069054. https://doi.org/10.1101/2020.04.29.069054. (2020).

30. Trucchi, E. et al. Unveiling diffusion pattern and structural impact of the most invasive SARS-CoV-2 spike mutation. bioRxiv 2020.05.14.095620. https://doi.org/10.1101/2020.05.14.095620. (2020).

31. Chu, H. et al. Comparative tropism, replication kinetics, and cell damage profiling of SARS-CoV-2 and SARS-CoV with implications for clinical manifestations, transmissibility, and laboratory studies of COVID-19: an observational study. Lancet Microbe 1, e14-e23 (2020).

32. Menzies, S. A. et al. The sterol-responsive RNF145 E3 ubiquitin ligase mediates the degradation of HMG-CoA reductase together with gp78 and Hrd1. eLife 7, e40009 (2018).

33. Daly, J. L. et al. Neuropilin-1 is a host factor for SARS-CoV-2 infection. bioRxiv 2020.06.05.134114. https://doi. org/10.1101/2020.06.05.134114. (2020).

34. Ibrahim, I. M., Abdelmalek, D. H., Elshahat, M. E. \& Elfiky, A. A. COVID-19 spike-host cell receptor GRP78 binding site prediction. J. Infect. 80, 554-562 (2020).

35. Katz, D. H. et al. Proteomic Profiling in Biracial Cohorts Implicates DC-SIGN as a Mediator of Genetic Risk in COVID-19. medRxiv 2020.06.09.20125690. https://doi.org/10.1101/2020.06.09.20125690. (2020).

36. Li, Y. et al. The MERS-CoV receptor DPP4 as a candidate binding target of the SARS-CoV-2 spike. iScience 23, 101160 (2020).

37. Ulrich, H. \& Pillat, M. M. CD147 as a target for COVID-19 treatment: suggested effects of azithromycin and stem cell engagement. Stem Cell Rev. Rep. https://doi.org/10.1007/s12015-020-09976-7 (2020).

38. Zhou, H. et al. Potential therapeutic targets and promising drugs for combating SARS-CoV-2. Br. J. Pharmacol. 177, 3147-3161 (2020).

39. Sardu, C. et al. Hypertension, thrombosis, kidney failure, and diabetes: is COVID-19 an endothelial disease? A comprehensive evaluation of clinical and basic evidence. J. Clin. Med. 9, 1417 (2020).

40. Debuc, B. \& Smadja, D. M. Is COVID-19 a new hematologic disease?. Stem Cell Rev. Rep. https://doi.org/10.1007/s12015-020-09987 $-4(2020)$.

41. Helal, M. A. et al. Molecular basis of the potential interaction of SARS-CoV-2 spike protein to CD147 in COVID-19 associatedlymphopenia. J Biomol Struct Dyn 1-11 https://doi.org/10.1080/07391102.2020.1822208.

42. Zamorano Cuervo, N. \& Grandvaux, N. ACE2: evidence of role as entry receptor for SARS-CoV-2 and implications in comorbidities. eLife 9 , e61390 (2020).

43. Muramatsu, T. Basigin (CD147), a multifunctional transmembrane glycoprotein with various binding partners. J Biochem 159, 481-490 (2016).

44. Wölfel, R. et al. Virological assessment of hospitalized patients with COVID-2019. Nature 581, 465-469 (2020).

45. Gubernatorova, E. O., Gorshkova, E. A., Polinova, A. I. \& Drutskaya, M. S. IL-6: relevance for immunopathology of SARS-CoV-2. Cytokine Growth Factor Rev. 53, 13-24 (2020).

46. Huertas, A. et al. Endothelial cell dysfunction: a major player in SARS-CoV-2 infection (COVID-19)?. Eur. Respir. J. https://doi. org/10.1183/13993003.01634-2020 (2020).

47. Latini, A. et al. COVID-19 and Genetic Variants of Protein Involved in the SARS-CoV-2 Entry into the Host Cells. Genes 11, 1010 (2020).

48. Yao, H. et al. Important functional roles of basigin in thymocyte development and T cell activation. Int. J. Biol. Sci. 10, 43-52 (2013).

49. Lucas, C. et al. Longitudinal analyses reveal immunological misfiring in severe COVID-19. Nature 584, 463-469 (2020).

50. Wood, L. \& Wright, G. J. High-content imaging for large-scale detection of low-affinity extracellular protein interactions. SLAS DISCOVERY: Adv. Sci. Drug Discov. 24, 987-999 (2019).

51. Kirk, P. et al. CD147 is tightly associated with lactate transporters MCT1 and MCT4 and facilitates their cell surface expression. EMBO J 19, 3896-3904 (2000).

52. Crosnier, C., Staudt, N. \& Wright, G. J. A rapid and scalable method for selecting recombinant mouse monoclonal antibodies. BMC Biol. 8, 76 (2010). 
53. Sun, Y., Gallagher-Jones, M., Barker, C. \& Wright, G. J. A benchmarked protein microarray-based platform for the identification of novel low-affinity extracellular protein interactions. Anal. Biochem. 424, 45-53 (2012).

54. Kerr, J. S. \& Wright, G. J. Avidity-based extracellular interaction screening (AVEXIS) for the scalable detection of low-affinity extracellular receptor-ligand interactions. J. Vis. Exp. https://doi.org/10.3791/3881 (2012).

55. Parrott, M. B. \& Barry, M. A. Metabolic biotinylation of secreted and cell surface proteins from mammalian cells. Biochem. Biophys. Res. Commun. 281, 993-1000 (2001).

56. Esposito, D. et al. Optimizing high-yield production of SARS-CoV-2 soluble spike trimers for serology assays. Protein Expr. Purif. https://doi.org/10.1016/j.pep.2020.105686 (2020).

57. Patterson, E. I. et al. Methods of inactivation of SARS-CoV-2 for downstream biological assays. bioRxiv (2020) https://doi. org/10.1101/2020.05.21.108035.

58. Finak, G., Jiang, W. \& Gottardo, R. CytoML for cross-platform cytometry data sharing. Cytometry A 93, 1189-1196 (2018).

\section{Acknowledgements}

pCG1-SARS-2-S was a kind gift from Stefan Pöhlmann (German Primate Centre, University of Göttingen). SARS-CoV-2/human/Liverpool/REMRQ0001/2020 was a kind gift from Lance Turtle (University of Liverpool) and David Matthews and Andrew Davidson (University of Bristol). Sheep anti-SARS-CoV-2 nucleocapsid antibody (DA114) was a kind gift from Paul Davies (obtained from MRC PPU Reagents and Services, University of Dundee). JS and GJW were funded by the Wellcome Trust Grant 206194. TWMC, EJDG, and PJL were supported by the Wellcome Trust (PRF 210688/Z/18/Z). This work was partly funded by UKRI/NIHR through the UK Coronavirus Immunology Consortium (UK-CIC).

\section{Author contributions}

J.S. performed all experiments except the CaLu-3 assays. T.W.M.C. and E.J.D.G. performed all CaLu- 3 infection assays. J.S. analyzed all data and wrote the manuscript. G.J.W. and P.J.L. supervised the research and edited the manuscript.

\section{Competing interests}

The authors declare no competing interests.

\section{Additional information}

Supplementary Information The online version contains supplementary material available at https://doi. org/10.1038/s41598-020-80464-1.

Correspondence and requests for materials should be addressed to J.S. or G.J.W.

Reprints and permissions information is available at www.nature.com/reprints.

Publisher's note Springer Nature remains neutral with regard to jurisdictional claims in published maps and institutional affiliations.

(c) (1) Open Access This article is licensed under a Creative Commons Attribution 4.0 International License, which permits use, sharing, adaptation, distribution and reproduction in any medium or format, as long as you give appropriate credit to the original author(s) and the source, provide a link to the Creative Commons licence, and indicate if changes were made. The images or other third party material in this article are included in the article's Creative Commons licence, unless indicated otherwise in a credit line to the material. If material is not included in the article's Creative Commons licence and your intended use is not permitted by statutory regulation or exceeds the permitted use, you will need to obtain permission directly from the copyright holder. To view a copy of this licence, visit http://creativecommons.org/licenses/by/4.0/.

(C) The Author(s) 2021 